\title{
Influence of poloxamer 407 on fractional and subfractional composition of serum lipoproteins of mice
}

\author{
Tatyana A. Korolenko ${ }^{1 *}$, Fedor V. Tuzikov ${ }^{2,3}$, Thomas P. Johnston ${ }^{4}$, Natalia A. Tuzikova ${ }^{2,3}$ \\ Elena E. Filjushina ${ }^{1}$, Viktoriya M. Loginova ${ }^{1}$, Natalia G. Savchenko $^{1}$

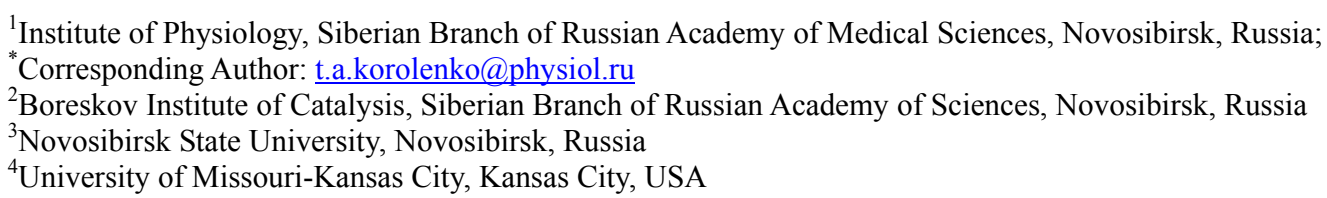

Received 4 March 2010; revised 22 March 2010; accepted 25 March 2010.

\begin{abstract}
Using a novel small-angle X-ray scattering (SAXS) method for determination of fractional and subfractional composition of lipoproteins (LPs), a significant elevation of total cholesterol-lipoproteins (C-LP) and, especially, total triglyceridelipoproteins (TG-LP), was shown in this work. Among the LP fractions, poloxamer 407 was shown to significantly increase proatherogenic total C-LDL, TG-LDL and, especially, their precursors C-VLDL and TG-VLDL, while only exhibiting a moderate increase in the antiatherogenic C-HDL and TG-HDL fractions. With regard to the VLDL subfractions, significant elevations were observed in both subfractions studied; namely, C-VLDL ${ }_{1-2}$ and C-VLDL ${ }_{3-5}$. Similar changes were noted in the TG-VLDL ${ }_{1-2}$ and TG- VLDL ${ }_{3-5}$ subfractions. The C-IDL and TG-IDL subfractions were increased significantly $(20-$ to 30 fold), while the $C-L L_{1-3}$ subfraction was moderately ( $\sim 3-$ to 5-fold) increased at $48 \mathrm{hrs}$ and at day 4 . In the moderately elevated ( 2- to 4-fold) anti-atherogenic HDL fraction, the $\mathrm{C}-\mathrm{HDL}_{2}$ subfraction was increased more significantly ( 4fold) compared to the $\mathrm{C}-\mathrm{HDL}_{3}$ subfraction; however, both C-HDL subfractions returned to baseline by day 4 . The elevation in the TG-HDL 2 subfraction was observed only at $\mathbf{2 4}$ hrs. Mouse models of hyperlipidemia and atherosclerosis are useful to evaluate the role of "individual" LPs, as well as their fractions and subfractions, in hyperlipidemia and the genesis of atherosclerosis.
\end{abstract}

Keywords: Poloxamer P-407; Dyslipidemia;
Serum Lipoprotein Fractions and Subfractions

\section{INTRODUCTION}

Changes of different classes of circulating lipoproteins are the important indicies of lipid metabolism in physiology and pathology; lipoproteins have been shown to also play a regulatory role in vivo [1,2]. The main lipoprotein classes consist of pro-atherogenic low-density lipoproteins (LDL), very-low-density lipoproteins (VLDL), and anti-atherogenic high density lipoproteins (HDL), and are widely used as common lipid biomarkers in atherosclerosis [1]. However, the biological role of subclasses of lipoproteins is still under investigation. The regulatory role of lipoproteins has been shown to relate to many intracellular processes, primarily to plasma membrane permeability and fluidity as the result of changes in the concentration of plasma membrane cholesterol, with subsequent modifications of receptors and transmembrane proteins (transmitters). In this process, the role of total HDL and HDL subfractions have especially high importance in connection with their unique capacity to accept cholesterol from the plasma membrane and transport them to other classes of lipoproteins. Hyperlipidemia is one of the main risk factors in the development of cardiovascular and cerebrovascular diseases common in contemporary society. In addition to the elevation of plasma LDL-cholesterol and VLDLcholesterol, hypertriglyceridemia is suggested to be an additional independent risk factor for atherosclerosis and coronary heart disease [3]. According to recent data, atherosclerosis is not only a metabolic lipid disease, but has also been considered to result from inflammation (chronic inflammatory disease). Statins have been reported to not only lower LDL by inhibiting the activity 
of 3-hydroxy-3-methylglutaryl coenzyme A (HMG-CoA) reductase, but also by reducing inflammation to endothelial cells.

For the prevention of atherosclerosis, it is important to study the mechanisms of the early effects of hyperlipidemia on different cell types involved in the pathogenesis of atherosclerosis. Therefore, it is necessary to know the types of lipoproteins involved in hyperlipidemia at the early stages of atherosclerosis. It is well known that during the process of atherosclerosis, foam cells initially trap the oxidized LDL molecules via scavenger receptors. The oxidized LDL molecule is digested and transformed, and is then presented to $\mathrm{T}$ lymphocytes, initiating the classic immunological reaction, and subsequently stimulates the inflammatory response. Formation of lipid-laden cells (mainly smooth muscle cells and macrophages) is followed by increased secretion of pro-inflammatory cytokines (like IL-6) and other factors (several types of matrix metalloproteases), which promote inflammation. Statin treatment has a pleiotropic protective effect, not only by inhibiting HMG-CoA reductase, but also by exerting its anti-inflammatory action.

The changes of serum lipoprotein levels responsible for pro- and anti-atherogenic action have been studied earlier [2]. However, recently, with help of new methods for characterizing different lipoprotein fractions and subfractions, some new data have been obtained on their role in the pathogenesis of atherosclerosis [4]. For this reason, the investigation of experimental models of hyperlipidemia is useful.

Poloxamer 407 is a block copolymer comprised of polyoxyethylene and polyoxypropylene units, which is known for its biocompatibility and potential to deliver different medications for a variety of disease states $[5,6]$. Following acute administration, poloxamer 407 was shown to induce significant hyperlipidemia, a model which has been used for testing several hypolipidemic drugs (statins, fibrates, and nicotinic acid) [7-11]. With chronic administration of poloxamer 407 (4 months) to mice, fibrofatty aortic lesions are developed, which are similar in size and number to those observed in classic diet-induced mouse models of atherogenesis [12,13].

Hyperlipidemia in acute poloxamer 407-induced administration to rodents was characterized by a dramatic elevation of both plasma cholesterol and triglycerides, which is usually not observed in patients with atherosclerosis. In the poloxamer 407 mouse model of atherosclerosis, the mechanism of cholesterol and TG elevation is associated with inhibition of cholesterol $7 \alpha$-hydroxylase and lipoprotein lipase, respectively [13], and not dependent on PPAR $\alpha$ [14]. It was also shown that a single injection of poloxamer 407 administration to mice caused hypercholesterolemia by inducing transient cho- lesterolgenesis and down-regulating low-density lipoprotein receptor expression [6]. The mechanism of elevation of different classes and, especially subfractions of lipoproteins with pro- and anti-atherogenic effects, is still not known.

Small-angle X-ray scattering (SAXS) is a small-angle scattering technique where the elastic scattering of X-rays (wavelength 0.1 to $0.2 \mathrm{~nm}$ ) by a sample, which has inhomogeneities in the nanometer range, is recorded at very low angles. This angular range contains information about the shape and size of macromolecules, characteristic distances of partially ordered materials, pore sizes, and other data. SAXS is capable of delivering structural information of macromolecules between 5 and $25 \mathrm{~nm}$, of repeat distances in partially ordered systems of up to $150 \mathrm{~nm}$. SAXS is used in the characterization of various materials. In the case of biologic macromolecules, such as proteins, the advantage of SAXS, over crystallography, is that a crystalline sample is not needed. The materials can be solid or liquid and they can contain solid, liquid, or gaseous domains (so-called particles) of the same or another material in any combination. SAXS is accurate, non-destructive, and usually requires only a minimum of sample preparation.

The aim of the present investigation was to evaluate the lipoprotein-cholesterol and lipoprotein-triglyceride fractions and subfractions in hyperlipidemia induced by a single dose of poloxamer 407 to mice. Our overarching goal was to quantify the changes in the serum lipoprotein levels after poloxamer 407 treatment by utilizing a novel technique; specifically, small-angle X-ray scattering (SAXS).

\section{MATERIALS AND METHODS}

\subsection{Materials}

Male CBA/C57BL mice (breeding station of the Institute of Cytology and Genetics, Russian Academy of Sciences, Novosibirsk, Russia) having a body mass of 20-25 g were used. Poloxamer P-407 (Pluronic F-127, Sigma) was administered to mice, as a single, i.p. injection, in a dose of $1000 \mathrm{mg} / \mathrm{kg}$. The animals were decapitated at 3 , 24, $48 \mathrm{hrs}$, and then at 4, 5, 7, 12, 14 days after a single dose of poloxamer 407. All experiments followed the official "Rules for the work involving experimental animals" and "Ethical Committee Recommendations of working with laboratory animals".

Serum was obtained after centrifugation of blood samples at $3000 \times \mathrm{g}$ for $15 \mathrm{~min}$ at $4^{\circ} \mathrm{C}$ (Eppendorf Centrifuge $5415 \mathrm{R}$, Germany) and stored at $-70^{\circ} \mathrm{C}$ until analysis of total cholesterol (C), triglycerides (TG) of lipoproteins (LP): C-LP, TG-LP, their fractions, and subfrac- 
tions.

\subsection{Small-Angle $X-R a y$ Scattering (SAXS)}

According to Otvos et al. (2002) [15], LP fractions were divided into the following 4 main classes: high density LP (HDL), low-density LP (LDL), very-low-density LP (VLDL), and chylomicrons (the last ones were not determined by the method used) or 7 subfractions: $\mathrm{HDL}_{3}$, $\mathrm{HDL}_{2}$, LDL, Intermediate-Density LP-IDL, VLDL $3-5$, VLDL $_{1-2}$, chylomicrons. Interval borders of fractions and subfractions (according to scale of sizes, $r_{0}$ ) were the same as was indicated by Otvos (2002) [15].

A novel method for determination of fractional composition of LPs using the small-angle X-ray scattering approach (SAXS) was used [4]. This method is inexpensive, quick, and capable of determining the relative content of different LP fractions, both as a size distribution of various LP particles and as absolute units of the total concentration of lipid in LP fractions. At the same time, clinical diagnostic laboratories usually determine the concentrations of individual lipids in LP fractions, mainly TG and free cholesterol (FC), and often the total concentration of TG, FC and high density lipoproteins (HDL) free cholesterol.

Measurement of fractional and subfractional composition of serum LP fractions and subfractions.was provided according to a method described earlier [4]. SAXS roentgenograms were obtained using a Siemens diffractometer (Germany) by the method of step-by-step scanning with use of a goniometer and X-ray scintillation detector. Small-angle roentgenograms were measured in the angular range: $h=0.013 \div 0.22 \mathrm{~A}^{-1}$, where $\mathrm{h}=4 \pi$. $\sin (\theta) / \lambda ; 2 \theta$-scattering angle. A special thermostatted $\left(20^{\circ} \mathrm{C}\right)$ quartz capillary cuvette $(0.6 \mathrm{~mm}$ in diameter $)$, having a wall thickness of $0.01 \mathrm{~mm}$ was used. The radiation wavelength $(\lambda)$ was $1.54 \mathrm{E}$. The small-angle X-ray roentgenograms were corrected by taking into account background scattering, adsorption, and collimation, after which the X-ray data became smoothed. The first step of mathematical processing of the SAXS data and computation checks of functions for size distribution of spherical particles were executed using a special computer program and algorithms described earlier, and also by use of optimization programs [4].

The results are reported as the mean \pm standard deviation of at least 3 different experiments for each sample analyzed. The differences between samples were analyzed by the Student's $t$-test, and a $\mathrm{P} \leq 0.05$ was considered statistically significant.

\subsection{Morphological Study of Liver}

For morphometrical study of liver tissue, samples were first fixed in a mixture of $2 \%$ paraformaldehyde and
$2.5 \%$ glutaraldehyde on $0.1 \mathrm{M}$ phosphate buffer, postfixed in $1 \%$ osmium tetroxide solution, and then embedded in an Epon-Araldit mixture. Semithin, 1 micron tissue sections were obtained using the Ultramicrotome LKB-8800-V (Bromma, Vallingby, Sweden), stained with toludine blue and examined under a STAR Zeiss light microscope (Germany). The numeric density of macrophage cells was calculated as the number of cells per 1 $\mathrm{MM}^{2}$ of tissue at a final magnification of 640 . Not less than 50 fields of view were studied for every series of experiments. The cell measurements were made with the Motic Images 2000 Program. Ultrastructural changes of liver cells were investigated with an electron microscope, JEM 1400 (Jeol, Japan). Quantitative data were then processed using the Statistica-4 program, and the reliability of distinctions judged by the Student's $t$-test.

\section{RESULTS}

The relative weight of liver and spleen increased $48 \mathrm{hrs}$ after poloxamer 407 administration (when the most significant hyperlipidemia was noted) (Figure 1). In peripheral blood, there was an increased number of monocytes observed at day 12 (Figure 2).

\subsection{Morphological Studies}

Light and electron microscopic studies demonstrated that liver sinusoids were considerably extended 1 day after poloxamer 407 administration, and that the numeric density of macrophages was significantly increased (almost twice) (Table 1). The heterogeneity of macrophage dimensions was strongly increased. Very large cells, with

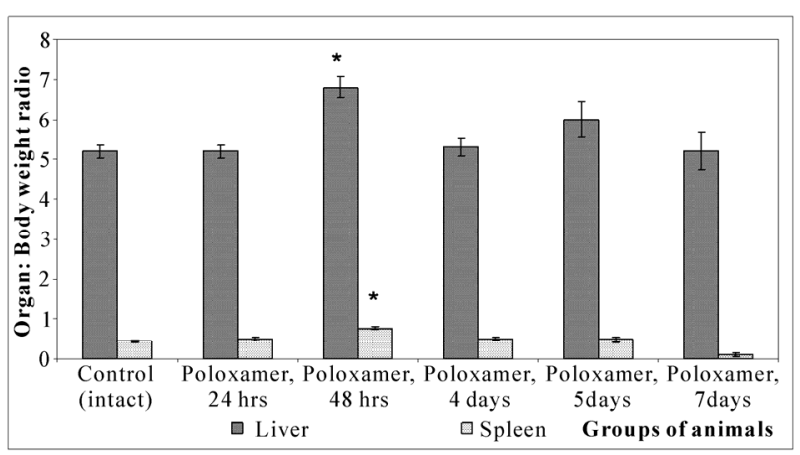

X-axis: time after the single administration of poloxamer 407 (1000 $\mathrm{mg} / \mathrm{kg}$ ) to mice

Y-axis: Organ: Body weight ratio of liver and spleen, $\%$.

The relative weight was expressed as the organ: body weight ratio Control group $n=26$, poloxamer-treated groups the mean \pm SEM represent a minimum of 7 mice at each time point.

The data are shown as mean $\pm \operatorname{SEM}\left({ }^{*} \mathrm{p}<0.01\right.$ vs control $)$

Figure 1. Relative liver and spleen weight of mice during single administration of poloxamer $407(1000 \mathrm{mg} / \mathrm{kg})$ to mice. 


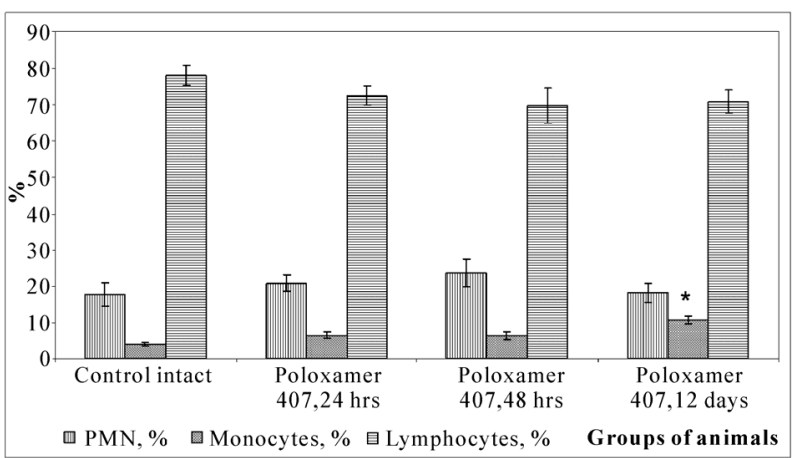

$\mathrm{X}$-axis: Time after poloxamer 407 administration to mice.

Y-axis: The number of PMN, monocytes and lymphocytes is expressed as $\%$ from the total amount of leukocytes.

Poloxamer was administered i.p. as a single dose $(1000 \mathrm{mg} / \mathrm{kg})$.

The number of animals in each group is 5 .

The data are shown as mean $\pm \operatorname{SEM}(* \mathrm{p}<0.05$ vs control).

Figure 2. Influence of poloxamer 407 on leukocytes composition of the peripheral blood of mice (\%).

Table 1. The numerical density of liver macrophages during single administration of poloxamer 407 to mice.

\begin{tabular}{cc}
\hline Groups, the number of mice & $\begin{array}{c}\text { The numeric density of liver } \\
\text { macrophages, per } \mathrm{mm}^{2}\end{array}$ \\
\hline 1. Control (intact) (5) & $1277.4 \pm 36.86$ \\
2. Poloxamer, 24 hrs (5) & $2002.6 \pm 36.58$ \\
& $\mathrm{P}<0.01$ \\
3. Poloxamer, 5 days after (5) & $844.6 \pm 28.74$ \\
& $\mathrm{P}<0.01$ \\
\hline
\end{tabular}

The data are shown as mean \pm SEM.

The number of mice is in the parentheses.

a cross-sectional area more than 90-100 square microns, were observed among macrophages, which comprised about $40 \%$ of the macrophage population. These macrophages were filled with granular material, so their cytoplasm had a foamy appearance (Figure 3).

Five days after poloxamer 407 administration, morphometric data for the liver demonstrated that the specific numerical density of macrophages was decreased, not only in comparison with the previous value, but in relation to the controls as well (Table 1). As before, large macrophages with a "foamy" cytoplasm containing electron light (transparent) material (possibly, of lipid origin) were observed, but the fraction of these cells was considerably reduced compared to the number of these cells in the previous experiment (Table 1). The cytoplasm of hepatocytes in most cases was loose and mostly crumbly, with several large vacuoles, and their size was moderately increased.

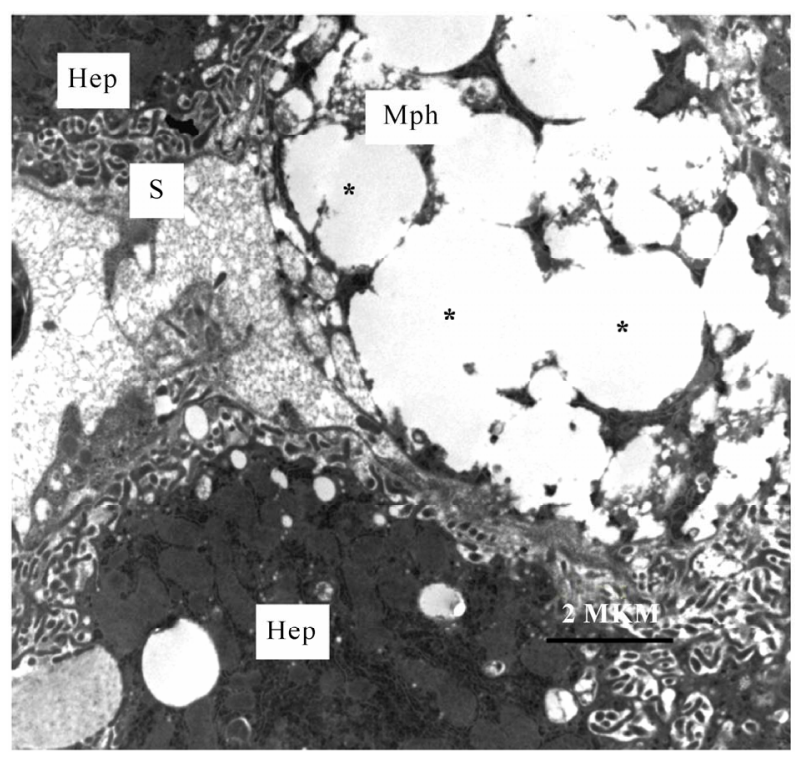

Electron micrograph of liver cells. In sinusoid lumen a large liver macrophage with foamy cytoplasm, filled by numerous electron light granules.

Denotes: Mph-macrophage; Hep-hepatocyte; S-sinusoid

*-electron light granules of macrophages

Poloxamer was administered i.p. as a single dose $(1000 \mathrm{mg} / \mathrm{kg})$.

Study was performed at the $1^{\text {st }}$ day after administration of a single dose of poloxamer 407 .

Figure 3. Influence of poloxamer 407 on ultrastructure of liver cells.

\subsection{Effect of Poloxamer 407 on Fractional and Subfractional Composition of Serum lipoproteins}

\subsubsection{Lipoprotein-Cholesterol (C-LP)}

Compared to intact (Control) mice, there was a significant ( $\sim 6$-fold) increase in the serum total C-LP at 24 and $48 \mathrm{hrs}$, as well as day 4 , in mice which were administered poloxamer 407 (Table 2). As shown in Table 2, among the total C-LP, the greatest increase ( 50 -fold) was observed with the pro-atherogenic C-VLDL fraction (both $\mathrm{C}-\mathrm{VLDL}_{1-2}$ and $\mathrm{C}-\mathrm{VLDL}_{3-5}$ subfractions), as well as with intermediate density lipoproteins (C-IDL) ( 20fold). However, it should be noted that serum concentrations of each fraction begin to decrease by day 4 . The C-LDL concentration increased about twice, relative to controls, and continued to remain elevated at day 4 . C-LDL ${ }_{1-3}$ was moderately ( 3 - to 5-fold) increased at 48 hrs and at day 4 (Table 2). The C-IDL and TG-IDL subfractions were increased significantly ( 20-30-fold), while the $\mathrm{C}-\mathrm{LDL}_{1-3}$ subfraction was moderately increased ( 3- to 5-fold) at $48 \mathrm{hrs}$ and at day 4 .

Among anti-atherogenic fractions and subfractions, only a moderate increase of C-HDL was noted ( 2- to 
Table 2. Concentration of C-LP fractions and subfractions (mg/dl) in murine serum during administration of poloxamer 407 (1000 $\mathrm{mg} / \mathrm{kg})$.

\begin{tabular}{|c|c|c|c|c|}
\hline LP & Control, intact $(\mathrm{n}=10)$ & Poloxamer, $24 \mathrm{hrs}(\mathrm{n}=10)$ & Poloxamer, 48 hrs $(\mathrm{n}=8)$ & Poloxamer, 4 days $(\mathrm{n}=5)$ \\
\hline C-LP (All) & $107.4 \pm 8.84$ & $662.9 \pm 82.40^{* * *}$ & $599.7 \pm 104.11^{* *}$ & $727.2 \pm 87.2^{*}$ \\
\hline C-VLDL & $6.5 \pm 1.85$ & $345.8 \pm 46.90^{* * *}$ & $271.3 \pm 73.02^{* *}$ & $134.4 \pm 46.23^{* *}$ \\
\hline${\mathrm{C}-\mathrm{VLDL}_{1-2}}$ & $0.3 \pm 0.07$ & $16.7 \pm 2.13^{* * *}$ & $12.8 \pm 4.70^{* *}$ & $8.9 \pm 4.11$ \\
\hline $\mathrm{C} \mathrm{VLDL}_{3-5}$ & $6.0 \pm 1.71$ & $329.1 \pm 45.54^{* * *}$ & $258.5 \pm 70.44^{* *}$ & $125.5 \pm 42.43^{* *}$ \\
\hline C-LDL & $43.1 \pm 7.67$ & $106.9 \pm 24.15^{* *}$ & $201.0 \pm 34.38^{* * *}$ & $247.3 \pm 31.52^{* * *}$ \\
\hline C-IDL & $1.9 \pm 1.61$ & $40.3 \pm 11.13^{* *}$ & $62.6 \pm 14.88^{* * *}$ & $34.9 \pm 13.32^{*}$ \\
\hline C-LDL ${ }_{1-3}$ & $41.2 \pm 8.61$ & $66.6 \pm 25.54$ & $146.1 \pm 32.68^{* *}$ & $212.4 \pm 35.56^{* *}$ \\
\hline C-HDL & $57.9 \pm 9.75$ & $210.2 \pm 48.36^{* *}$ & $127.4 \pm 41.93$ & $45.4 \pm 13.30$ \\
\hline C-HDL 2 & $43.1 \pm 10.02$ & $166.5 \pm 38.70^{* *}$ & $91.9 \pm 47.31$ & $16.9 \pm 8.55$ \\
\hline C-HDL ${ }_{3}$ & $14.7 \pm 3.55$ & $43.7 \pm 20.21$ & $35.5 \pm 8.94^{*}$ & $28.5 \pm 9.23$ \\
\hline
\end{tabular}

The data are shown as mean $\pm \operatorname{SEM}\left({ }^{*} \mathrm{p}<0.05 ;{ }^{* *} \mathrm{p}<0.01 ;{ }^{* * *} \mathrm{p}<0.001\right.$ vs control $)$

The number of animals is in the parentheses.

Abbreviations: C-LP-cholesterol of lipoproteins; C-HDL-cholesterol of high density lipoproteins; C-LDL-cholesterol of low density lipoproteins; C-IDL-cholesterol of intermediate density lipoproteins; C-VLDL-cholesterol of very low density lipoproteins.

4-fold), with a return to baseline by day 4 (primarily due to a decrease in the concentration of the $\mathrm{C}-\mathrm{HDL}_{2}$ subfraction), while the $\mathrm{C}-\mathrm{HDL}_{3}$ subfraction showed a moderate increase (Table 2).

\subsubsection{Lipoproteins-TG (TG-LP)}

In general, the total amount of TG-LP increased more significantly as compared to C-LP ( 20-fold), with a tendency to return to normal levels by day 4 (Tables 2 and 3). TG-VLDL "behavior" was similar to C-VLDL (increased 20- to 40-fold), and both subfractions (TGVLDL $_{1-2}$ and $T G-V_{L D L}$ ) were responsible for the elevation of the TG-VLDL fraction (Tables 2 and 3). Similar to C-LDL, an increase in TG-LDL was observed ( 6- to 8-fold). The level of C-IDL (Table 2) and TG-IDL (Table 3) was shown to be increased ( $18-$ to 28 -fold, respectively) with a significant difference at 24 , $48 \mathrm{hrs}$, and on day 4, as compared to the controls ( Tables 2 and 3). A moderate elevation was also observed for C-HDL and TG-HDL ( 2- to 3-fold) by $24 \mathrm{hrs,} \mathrm{but}$ serum levels were not significantly greater than controls from 24 hrs onward (Tables 2 and 3). The TG-HDL 2 subfraction was elevated only at $24 \mathrm{hrs}$ after poloxamer 407 administration when compared to controls (Table 3). There were no significant changes of the TG-HDL $\mathrm{TL}_{3} \mathrm{sub}$ fraction (Table 3).

\section{DISCUSSION}

Atherosclerosis is a complex disorder of lipid metabo- lism and a chronic inflammatory disease [16]. Changes in lipoprotein metabolism and hyperlipidemia play an important role in the progression of atherosclerosis. The initial period of atherosclerosis development and the interaction of lipoproteins with cells involved in the dislipidemic state merits further investigation. Mouse models of hyperlipidemia and atherosclerosis are useful tools with which to study the role of "individual" lipoproteins of different classes.

Lipoproteins are divided into 4 main fractions (classes) -HDL, LDL, VLDL, and chylomicrons, or 7 subfractions $\left(\mathrm{HDL}_{3}, \mathrm{HDL}_{2}, \mathrm{LDL}, \mathrm{IDL}, \mathrm{VLDL}_{3-5}, \mathrm{VLDL}_{1-2}\right.$, chylomicrons), or $16 \mathrm{sub} / \mathrm{subfractions}[15,17,18]$. Most serum cholesterol, about $60-70 \%$ of total serum cholesterol, is known to be contained in LDL-C as the major atherogenic lipoprotein fraction [1]. During the last decade, several new methods, including the analytical ultracentrifugal micromethod, were developed for identification of atherogenic LDL, and their subclasses, without their preparative isolation [19].

At the same time, it is important to investigate other lipoprotein fractions - namely, VLDL and HDL, as well as their subfractions. VLDL, which is a precursor of LDL, and some forms of VLDL, is atherogenic, especially their remnant forms. VLDL contains most of the serum triglycerides, their role, of which, is now thought to be an independent and important risk factor in atherosclerosis [20]. HDL is known as an antiatherogenic fraction of lipoproteins [21]. With the assistance of the new physicochemical SAXS method, it is possible to simultaneously 
Table 3. Concentration of TG-LP fractions and subfractions (mg/dl) in murine serum during administration of poloxamer 407 (1000 $\mathrm{mg} / \mathrm{kg})$.

\begin{tabular}{|c|c|c|c|c|}
\hline LP fractions, Subfractions & Control, intact $(\mathrm{n}=10)$ & Poloxamer, $24 \mathrm{hrs}(\mathrm{n}=10)$ & Poloxamer, 48 hrs $(\mathrm{n}=8)$ & Poloxamer, 4 days $(\mathrm{n}=5)$ \\
\hline TG-LP (All) & $54.5 \pm 6.57$ & $1107.4 \pm 148.48^{* * *}$ & $902.2 \pm 223.69^{* *}$ & $517.3 \pm 152.67^{* *}$ \\
\hline TG-VLDL & $19.5 \pm 4.85$ & $952.1 \pm 129.69^{* * *}$ & $735.2 \pm 198.61^{* *}$ & $383.0 \pm 140.44^{* *}$ \\
\hline TG-VLDL $_{1-2}$ & $3.1 \pm 0.61$ & $148.7 \pm 26.59^{* * *}$ & $103.9 \pm 36.43^{* *}$ & $80.1 \pm 41.67$ \\
\hline TG-VLDL $_{3-5}$ & $16.0 \pm 4.30$ & $803.4 \pm 114.37^{* * *}$ & $631.3 \pm 175.49^{* *}$ & $302.9 \pm 101.23^{* *}$ \\
\hline TG-LDL & $14.0 \pm 1.85$ & $80.7 \pm 13.92^{* *}$ & $120.7 \pm 33.23^{* *}$ & $117.8 \pm 14.79^{* * *}$ \\
\hline TG-IDL & $2.6 \pm 2.15$ & $53.8 \pm 14.83^{* *}$ & $83.4 \pm 19.82^{* *}$ & $46.6 \pm 17.74^{*}$ \\
\hline TG-LDL $1-3$ & $11.4 \pm 2.31$ & $24.6 \pm 10.37$ & $47.5 \pm 11.14^{* *}$ & $71.2 \pm 15.19^{* *}$ \\
\hline TG-HDL & $21.2 \pm 3.58$ & $76.9 \pm 17.67^{* *}$ & $46.4 \pm 15.21$ & $16.5 \pm 5.00$ \\
\hline TG-HDL $_{2}$ & $15.9 \pm 3.68$ & $60.9 \pm 14.31^{* *}$ & $33.6 \pm 17.17$ & $6.4 \pm 3.16$ \\
\hline TG-HDL $_{3}$ & $5.3 \pm 1.25$ & $16.1 \pm 7.38$ & $12.8 \pm 3.14$ & $10.14 \pm 3.39$ \\
\hline
\end{tabular}

The data are shown as mean $\pm \operatorname{SEM}\left({ }^{*} \mathrm{p}<0.05 ;{ }^{* *} \mathrm{p}<0.01 ;{ }^{* * *} \mathrm{p}<0.001\right.$ vs control $)$

The number of animals is in the parentheses.

Abbreviations: TG-LP-triglycerides of lipoproteins; TG-HDL-triglycerides of high density lipoproteins; TG-LDL-triglycerides of low density lipoproteins; TG-IDL-triglycerides of intermediate density lipoproteins; TG-VLDL-triglycerides of very low density lipoproteins.

investigate all three major classes of lipoproteins (VLDL, LDL, HDL) and all subfractions, except chylomicrons (the particles are too large for this method of analysis) [4]. Moreover, it is known that fasting human plasma, as well as plasma of mice 12-14 hrs after feeding, contains negligible chylomicrons. The available physical-chemical data in the literature suggests the existence of a stable thermodynamic equilibrium between all lipoprotein types. This equilibrium is specific for each physiological state in the human and helps to stabilize the lipoprotein structure and assist with normal lipoprotein functioning. In the SAXS method, the dynamic equilibrium between different LP types was used as a physico-chemical basis, and a general model, which takes into account the relative amount of all LP types and their transitional forms (in terms of the relative size of LP particles and their relative concentrations) was developed [4].

As was shown in this work, the poloxamer 407-induced model of hyperlipidemia in mice was characterized by a significant elevation of both C-LP and TG-LP total fractions. Similar data were obtained by other investigators that studied the level of atherogenic and antiatherogenic lipoprotein fractions with other, more common methods [5]. It is necessary to mention that the concentration of pro-atherogenic LDL and VLDL fractions increased dramatically, as compared to a moderate increase in the level of HDL particles. This is probably one of the reasons why repeated administration of poloxamer 407 to mice successfully reproduces the atherosclerotic process in rodents, where atherosclerosis is difficult to develop due to the normally high concentration of antiatherogenic HDL. These data were supported by the observation that statin treatment in patients with cardiovascular diseases decreases the mortality by decreasing the C-LDL levels, thereby, exerting the protective lipid-lowering and also pleiotropic effects [2].

Elevated plasma concentration of C-LDL is considered a risk factor for atherosclerosis development. LDL, especially oxLDL particles, infiltrates the vascular wall and can be taken up by macrophages. This is followed by secretion of proinflammatory factors which are involved in the pathogenesis of atherosclerosis. HDL, like other fractions of lipoproteins (LDL and VLDL), are a heterogeneous group of lipoproteins; their core contains some free cholesterol, triglycerides, and cholesterol esters $[21,22]$. HDL particles are involved in reverse cholesterol transport, and their atheroprotective role may be connected with regulation of adhesion molecule expression and the prevention of oxidative modifications of LDL [16,21]. It was shown in this work that both subfractions of C-HDL were only moderately increased in poloxamer 407-treated mice and returned to normal levels by day 4 following poloxamer administration. Similar changes were observed in the TG-HDL fraction and subfractions.

In general, there are many factors influencing serum 
concentration of lipoproteins (biosynthesis, uptake by endocytosis, secretion, and degradation). The liver is the main source of VLDL. LDL catabolism occurs primarily in the liver and in most extrahepatic tissues [2]. Different types of cells have been shown to have LDL receptors on the plasma membrane [6]. Catabolism of LDL has been shown to occur inside of lysosomes [23,24]. Metabolism of HDL is closely related to homeostasis of lipids in the intact vertebrate, with lipoproteins residing in both the serum and in tissues [2].

Poloxamer 407 appears to act as a general lipase inhibitor. Mammalian lipases include three main lipase members; namely, lipoprotein lipase, hepatic lipase, and endothelial lipase, acting in vitro and in vivo on lipoproteins [23]. Lipoprotein lipase has a high triglyceride lipase activity, while endothelial lipase exerts phospholipase activity. Endothelial lipase is able to hydrolyze lipids from HDL, while hepatic lipase exerts functional activity on all classes of lipoproteins [23]. Lipoprotein lipase (EC 3.1.1.34) (LPL) is a key enzyme in lipoprotein metabolism. LPL belongs to the class of enzymes known as hydrolases, which catalyze the degradation of glycerides, including triglycerides. Poloxamer 407 (as well as the nonionic detergent Triton WR 1339) has been shown to inhibit LPL, and this effect has been suggested as playing a crucial role in disturbances of lipoprotein transport and a significant elevation in the serum levels of LP-TG

There are some similarities in the models of poloxamer and Triton WR 1339-induced hyperlipidemia following a single intraperitoneal administration of each agent. Both models were characterized by dramatic increased serum concentrations of cholesterol and, especially triglycerides, in rats and mice. As mentioned above, the resultant hypertriglyceridemia occurs due to surfactant-mediated inhibition of LPL's enzymatic activity $[25,26]$. Moreover, Triton WR 1339 is a well-known lysosomotropic agent, accumulating inside of lysosomes of liver cells; specifically, hepatocytes and Kupffer cells [27]. In both species of rodents, Triton-induced hyperlipidemia occurred 24 hrs after detergent administration, and was followed by a dramatic increase in both lipoprotein cholesterol and, especially, of lipoprotein triglyceride concentrations [26,27]. We have recently shown significant increases in the concentration of atherogenic C-LDL, C-VLDL (due to an increase of the $\mathrm{VLDL}_{3-5}$ subfraction), and IDL in mice, and even more profound elevations in rats (Korolenko et al., submitted In Press). These hyperlipidemic animal models can be used to study the role of lipoproteins, especially lipoprotein triglycerides, (but also C-LDL and C-VLDL) in the pathogenesis of atherosclerosis, as well as for testing the efficacy of new hypolipidemic drugs. The poloxamer 407-induced mouse model of hyperlipidemia would appear to resemble a Type $2 \mathrm{a} / 2 \mathrm{~b}$ or 3 dislipidemia in humans.

Mice and rat models of hyperlipidemia are used more often now because of their convenience, the ability to genetically alter the animals to evaluate the effect of a particular gene on lipid metabolism, and because they are cost-effective. The poloxamer 407-induced mouse model of dyslipidemia and atherosclerosis reliably reproduces the hyperlipidemic state, and, if administered on a repetitive basis (or, if dosed continuously with the aid of an implanted osmotic pump), causes formation of fibrofatty aortic lesions in the same size and number as classic diet-induced or gene-knockout mouse models [28].

Poloxamer 407 has been used in several fields of medicine as a nano-carrier for different drugs for the treatment of several inflammatory diseases and tumors [29]. However, when used to induce dyslipidemia and atherosclerosis in C57BL/6 mice, it must be emphasized that these are supraphysiologic doses of P-407 that would never be utilized in any commercial formulation employing its sustained-release or reverse-thermal gelation properties. Additionally, most applications of poloxamer 407 in the pharmaceutical literature deal with non-parenteral routes of delivery, unlike the intraperitoneal administration of poloxamer 407 to intentionally induce dyslipidemia in mice [30-34].

In conclusion, we have identified changes in the lipid fractions and subfractions following a single dose of poloxamer 407 to mice, as well as characterized the relative spleen and liver weights, and the effects on blood leukocytes. Additionally, we have pioneered the use of SAXS as a method to determine the concentrations of the serum lipids and lipid subfractions. This new analytical approach can now be successfully employed to determine the concentrations of lipoprotein fractions, and their subfractions, in either mouse or human plasma samples for a more complete picture of the hyperlipidemic state.

\section{ACKNOWLEDGEMENTS}

This work was partially supported by integrated grant of Siberian Branch of the Russian Academy of Medical Sciences and Far East Division of Russian Academy of Sciences on natural immunomodulators study (2006-2008). Authors are grateful to engineer M.Tuzikov for help in Small-angle X-ray scattering, Dr. Monoszon A., Dr. Kisarova Ya., Dr. Sukhovershin R., Dr. K. Loktev for technical help during preparing of the manuscript.

\section{REFERENCES}

[1] Anderson, L. (2005) Candidate-based proteomics in the 
search for biomarkers of cardiovascular disease. The Journal of Physiology, 563(1), 23-60.

[2] Gotto, A.M.Jr, and Farmer, J.A. (2006) Drug insight: The role of statins in combination with ezetimide to lower LDL cholesterol. Nature Clinical Practice Cardiovascular Medicine, 3(12), 664-672.

[3] Ugawa, T., Kakuta, H., Moritani, H., Inagaki, O. and Shikama, H. (2003) YM-53601, a novel squalene synthase inhibitor, suppresses lipogenic biosynthesis and lipid secretion in rodents. British Journal of Pharmacology, 139(1), 140-146.

[4] Tuzikov, F.V., Tuzikova, N.A., Galimov, R.V., Panin, L.E. and Nevinsky, G.A. (2002) General model to describe the structure and dynamic balance between different human serum lipoproteins and its practicl application. Medical Science Monitor, 8(6), pp. 79-88.

[5] Dumortier, G., Grossiord, J.L., Agnely, F. and Chaumeil, J.C. (2006) A review of poloxamer 407 pharmaceutical and pharmacological Characteristics. Pharmaceuticals Research, 23(12), 2709-2728.

[6] Leon, C., Wasan, K.M., Sachs-Barrable, K. and Johnston, T.P. (2006) Acute P-407 administration to mice causes hypercholesterolemia by inducing cholesterolgenesis and down-regulating low-density lipoprotein receptor expression. Pharmaceuticals Research, 23(7), 1597-1607.

[7] Kim, H.Y., Jeong, D.M., Jung, H.J., Jung, H.J., Yozokawa, T. and Choi, J.S. (2008) Hypolipidemic effects of Sophora Flavescens and its constituents in poloxamer 407-induced hyperlipidemic and cholesterol-fed rats. Biological \& Pharmaceutical Bulletin, 31(1), 73-78.

[8] Johnston, T.P. and Palmer, W.K. (1997) The effect of pravastatin on hepatic 3-hydroxy-3-methylglutaryl CoA reductase obtained from poloxamer 407-induced hyperlipidemic rats. Pharmacotherapy, 17(2), 342-347.

[9] Johnston, T.P., Baker, J.C., Hall, D., Jamal, S., Palmer, W.K. and Emeson, E.E. (2000) Regression of poloxamer 407-induced atherosclerotic lesions in C57BL/6 mice using atorvastatin. Atherosclerosis, 149(2), 303-313.

[10] Johnston, T.P., Nguyen, L.B., Chu, W.A. and Shefer, S. (2001) Potency of select statin drugs in a new mouse model of hyperlipidemia and atherosclerosis. International Journal of Pharmaceutics, 229(1-2), 75-86.

[11] Nash, V.J., Johnston, T.P. and Palmer, W.K. (1996) Effects of nicotinic acid on poloxamer 407-induced hyperlipidemia. Pharmacotherapy, 16(1), 10-15.

[12] Johnston, T.P., Li, Y., Jamal, A.S., Stechschulte, D.J. and Dileepan, K.N. (2003) Poloxamer 407-induced atherosclerosis in mice appears to be due to lipid derangements and not due to its direct effects on endothelial cells and macrophages. Mediators of Inflammation, 12(3), 147155.

[13] Johnston, T.P. (2009) Poloxamer 407 increases soluble adhesion molecules, ICAM-1, VCAM-1, and E-selectin, in C57BL/6 Mice. Journal of Pharmacy and Pharmacology, 61(12), 1681-1688.

[14] Johnston, T.P. and Waxman, D.J. (2008) The induction of atherogenic dyslipidemia in poloxamer 407-treated mice is not mediated through PPAR $\alpha$. Journal of Pharmaceutical Sciences, 60(6), 753-759.

[15] Otvos, J.D. (2002) Measurement of lipoprotein subclass profiles by nuclear magnetic resonance spectroscopy. Clinical Laboratory, 48(3-4), 171-180.
[16] Pelton, P.D, Patel, M. and Demarest, K.T. (2005) Nuclear receptors as potential targets for modulating reverse cholesterol transport, Current Topics in Medicinal Chemistry, 5(3), 265-282.

[17] Jeyarajah, E.J., Cromwell, W.C. and Otvos, J.D. (2006) Lipoprotein particle analysis by nuclear magnetic resonance spectroscopy. Clinics in Laboratory Medicine, 26(4), 847-870.

[18] Cromwell, W.C. and Otvos, J.D. (2006) Heterogeneity of low-density lipoprotein particle number in patients with type 2 diabetes mellitus and low-density lipoprotein cholesterol $<100 \mathrm{mg} / \mathrm{dl}$. American Journal of Cardiology, 98(12), 1599-1602.

[19] Bozóky, Z., Fülöp, L. and Köhidai, L. (2001) A short run new analytical ultracentrifugal micromethod for determining low-density lipoprotein sub-fractions using Schlieren refractometry. European Biophysics Journal, 29(8), 621-627.

[20] Barcia, A.M. and Harris, H.W. (2005) Triglyceride-rich lipoproteins as agents of innate immunity, Clinical Infectious Diseases, 41(10), 498-503.

[21] Tanaka, H., Ishida, T., Johnston, T.P., Yasuda, T., Ueyama, T., Kojima, Y., Kundu, R.K., Quertermous, T. and Hirata, K.-I. (2009) Role of endothelial lipase in plasma HDL levels in a murine model of hypertriglyceridemia. Journal of Atherosclerosis and Thrombosis, 16(4), 327-338.

[22] Gatica, L.V., Vega, V.A., Zirulnik, F., Oliveros, L.B. and Gimenez, M.S. (2006) Alterations in the lipid metabolism of rat aorta: Effects of vitamin A deficiency. Journal of Vascular Research, 43(6), 602-610.

[23] Brown, R.J. and Rader, D.J. (2007) Lipases as modulators of atherosclerosis in murine models. Current Drug Targets, 8(12), 1307-1319.

[24] Ballabio, A. and Gieselmann, V. (2009) Lysosomal disorders: From storage to cellular damage. Biochimica et Biophysica Acta, 1793(4), 684-696.

[25] Johnston, T.P. and Palmer, W.K. (1993) Mechanism of poloxamer 407-induced hypertriglyceridemia in the rat. Biochemical Pharmacology, 46(6), 1037-1042.

[26] Millar, J.S., Cromley, D.A, McCoy, M.G., Rader, D.J. and Billheimer, J.T. (2005) Determining hepatic triglyceride production in mice: Comparison of poloxamer 407 with triton WR 1339. Journal of Lipid Research, 46(9), 2023-2028.

[27] Schneider, P., Korolenko, T.A. and Busch, U. (1997) A review of drug-induced lysosomal disorders of the liver in man and laboratory animals. Microscopy Research and Technique, 36(4), 253-275.

[28] Johnston, T.P. (2004) The P-407-induced murine model of dose-controlled hyperlipidemia and atherosclerosis: A review of findings to date. Journal of Cardiovascular Pharmacology, 43(4), 595-606.

[29] Kuzman, D., Fon Tacer, K., Cerne, M., Rezen, T., Acimovic, J., Cegovnik, U., Kocjan, D., Urleb, U. and Rozman, D. (2009) Modulation of hepatic transcriptome in the poloxamer P-407 hyperlipidemia mouse model. Acta Chimica Slovenica, 56(1), 262-269.

[30] Fruchart, J.C. and Duriez, P. (2006) Mode of action of fibrates in the regulation of triglyceride and HDL-cholesterol metabolism. Drugs Today (Barc), 42(1), 39-64.

[31] Butler, J.A., Hagen, T.M. and Moreau, R. (2009) Lipoic acid improves hypertriglyceridemia by stimulating tria- 
cylglycerol clearance and downregulating liver triacylglycerol secretion. Archives of Biochemistry and Biophysics, 485(1), 63-71.

[32] Mora, S., Otvos, J.D., Rifai, N., Rosenson, R.S., Buring, J.E. and Ridker, P.M. (2009) Lipoprotein particle profiles by nuclear magnetic resonance compared with standard lipids and apolipoproteins in predicting incident cardiovascular disease in women. Circulation, 119(17), 931939.

[33] Zhou, X., Johnston, T.P., Johansson, D., Parini, P., Funa,

\section{ABBREVIATIONS}

C-cholesterol

TG-triglycerides

LP-lipoproteins

C (TG)-HDL-cholesterol (triglycerides) of high density lipoproteins

C (TG)-LDL-cholesterol (triglycerides) of low density
K., Svensson, J. and Hansson, G.K. (2009) Hypercholesterolemia leads to elevated TGF-beta 1 activity and T helper 3-dependent autoimmune responses in atherosclerotic mice, Atherosclerosis, 204(2), 381-387.

[34] Gotto, A.M. and Farmer, J.A. (2007) Atherosclerosis: Pathogenesis, morphology, and risk factors. In: Willerson J.T., Wellens H., Cohn J.N. and Holmes D.R.Jr. Eds., Cardiovascular Medicine, 3rd Edition, Springer, London, 1593-1613. lipoproteins

C (TG)-IDL-cholesterol (triglycerides) of intermediate density lipoproteins

C (TG)-VLDL-cholesterol (triglycerides) of very low density lipoproteins

SAXS-Small-angle X-ray scattering 